\title{
Enterovirus infection is associated with an increased risk of childhood type 1 diabetes in Taiwan: a nationwide population-based cohort study
}

\author{
Hsiao-Chuan Lin • Chung-Hsing Wang • Fuu-Jen Tsai • \\ Kao-Pin Hwang • Walter Chen • Cheng-Chieh Lin • \\ Tsai-Chung Li
}

Received: 21 May 2014 / Accepted: 4 September 2014 /Published online: 22 October 2014

(C) Springer-Verlag Berlin Heidelberg 2014

\begin{abstract}
Aims/hypothesis This study compared the incidence rate of type 1 diabetes in children diagnosed with enterovirus (EV) infections with that in age- and sex-matched children without EV infection in a population-based cohort. In addition, we examined whether the direction or magnitude of the association between EV infection and type 1 diabetes differs according to atopic disease status in children.
\end{abstract}

\section{H.-C. Lin}

Department of Public Health, College of Public Health,

China Medical University, Taichung, Taiwan

H.-C. Lin • C.-H. Wang • K.-P. Hwang • W. Chen • C.-C. Lin ( $\triangle)$ School of Medicine, College of Medicine, China Medical University, 91 Hsueh-Shih Road, Taichung 40402, Taiwan

e-mail: cclin@mail.cmuh.org.tw

H.-C. Lin $\cdot$ C.-H. Wang $\cdot$ F.-J. Tsai $\cdot$ K.-P. Hwang $\cdot$ W. Chen Department of Pediatrics, China Medical University Hospital, Taichung, Taiwan

F.-J. Tsai • C.-C. Lin

Department of Medical Research, China Medical University

Hospital, Taichung, Taiwan

F.-J. Tsai

College of Chinese Medicine, China Medical University,

Taichung, Taiwan

\section{C.-C. Lin}

Department of Family Medicine, China Medical University Hospital, Taichung, Taiwan

\section{T.-C. Li $(\bowtie)$}

Graduate Institute of Biostatistics, College of Management, China Medical University, 91 Hsueh-Shih Road, Taichung 40402, Taiwan e-mail: tcli@mail.cmu.edu.tw

\section{T.-C. Li}

Department of Healthcare Administration, College of Medical and Health Science, Asia University, Taichung, Taiwan
Methods We used insurance claims data from Taiwan's National Health Insurance Research Database to derive type 1 diabetes incidence in children aged up to 18 years with or without a diagnosis of EV infection during 2000-2008. Incidence rate ratios and HRs of type 1 diabetes for $\mathrm{EV}$ infection were estimated by Poisson regression and Cox's proportional hazard regression model.

Results Overall incidence of type 1 diabetes was higher in the EV than in the non-EV infection cohort (5.73 vs 3.89 per 100,000 person-years; incidence rate ratio 1.48 [95\% CI 1.19, 1.83]), with an adjusted HR of 1.48 (95\% CI 1.19, 1.83). Among children without EV, incidence increased with age at diagnosis of EV infection, except in those aged 5-10 years. The HRs of type 1 diabetes in children with allergic rhinitis, bronchial asthma or either one of these atopic diseases showed more variation than in those children without these diseases. Conclusions/interpretation This nationwide retrospective cohort study found a positive correlation between type 1 diabetes and EV infection. The results suggest that a preventive strategy, such as an effective vaccine against $\mathrm{EV}$ infection, may lessen the incidence of type 1 diabetes in Taiwan.

Keywords Enterovirus · National Health Insurance programme $\cdot$ Type 1 diabetes

\author{
Abbreviations \\ EV Enterovirus \\ ICD-9-CM ICD-9, Clinical Modification \\ NHIRD National Health Insurance Research Database
}

\section{Introduction}

Diabetes is one of the most common paediatric chronic diseases. Recent studies have shown a rapid global increase in 
childhood type 1 diabetes, particularly in children under 5 years of age [1-5]. Incidence varies greatly among ethnic groups: age-adjusted incidence in children ranges from 0.1 per 100,000 person-years in China and Venezuela [4] to 64.3 per 100,000 person-years in Finland [6]. In Taiwan, average yearly crude incidence was 3.75 per 100,000 in children aged 0-14 years during 1992-1996 [7].

Type 1 diabetes is caused by a complex interaction between genetic susceptibility, the immune system and environmental factors $[8,9]$. Although genetic predisposition to type 1 diabetes has been elucidated, the environmental triggers of the disease have not been proven, although evidence points to involvement of enterovirus (EV) infection (poliovirus, Coxsackievirus A, Coxsackievirus B and echovirus) [10]. Gamble et al were the first to report a possible link between $\mathrm{EV}$ infection and type 1 diabetes in 1969 [11, 12]. Evidence for the contribution of Coxsackievirus $\mathrm{B}$ infections in the pathogenesis of type 1 diabetes abounds [13-15], yet several other EVs species, including Coxsackievirus A [16-18], echoviruses [16, 17] and EVs of A-D species [19], may also have a role. Cases of new-onset type 1 diabetes occur in seasonal patterns [11,20], sometimes in clusters or small outbreaks, often peaking 1-2 months after high EV infection activity [21]. A recent study on intrafamilial spread of EV infections reported that $20 \%$ of siblings of diabetic probands acquired type 1 diabetes with a latency of 3-25 months [19]. Evidence for this association is supported by detection of EVs in the blood [13, 14, 17, 22], pancreas [15, 23-26] and gut mucosa [27] of patients with type 1 diabetes. Histopathologic evidence indicates that expression of enteroviral capsid protein VP1 in islet cells of patients with type 1 diabetes is related to induction of protein kinase $\mathrm{R}$ and downregulation of Mcl-1 [23, 24].

Despite more than 40 years of research, the role of EVs in type 1 diabetes is far from established; chief obstacles stem from difficulty in obtaining pancreatic tissue from patients $[25,28]$. A recent, large meta-analysis of observational molecular studies re-affirmed the association between human EV infection and type 1 diabetes [10]. Most of the molecular studies came from Western countries [10], Cuba [29], Australia [30], Egypt [31] and/or Japan [32]; none explained the rising tendency of the association between EV infection and type 1 diabetes in people of Han Chinese ethnicity [10]. In Finland, a prospective birth cohort study has suggested that Coxsackievirus B1 triggers the induction of $\beta$-cell autoimmunity that, in turn, heralds type 1 diabetes [13]. A case-control study involving a virus antibody survey has also alluded to an association between Coxsackievirus B1 and type 1 diabetes [14]. However, prior explorations of the association between EV infection and type 1 diabetes, including case-control and cohort studies, have been limited by small sample sizes. Besides EV infection, an inverse association has been found between atopic diseases and childhood type 1 diabetes [33].
To link EV infection and subsequent type 1 diabetes, our nationwide population-based cohort used a large-scale dataset available from Taiwan's National Health Insurance programme. The study aimed to compare the incidence rate of type 1 diabetes in children diagnosed with $\mathrm{EV}$ infections with that in age- and sex-matched children without EV infection. We also examined the interaction between EV infection and atopic diseases, a possible aetiologic factor.

\section{Methods}

Data sources A dataset of claims from the National Health Insurance Research Database (NHIRD) was obtained from Taiwan's National Health Insurance programme, a universal health insurance system implemented by the Department of Health as of March 1995 and now covering approximately $99 \%$ of the 23.74 million residents of Taiwan [34]. Data in the NHIRD includes all claims data for 2000-2008 for children aged 18 years or younger; these data were randomly sampled with a selection probability of 0.5 from the whole population of the same age, and comprehensive information was obtained about demographics, dates of clinical visits, diagnostic codes, details of prescriptions, expenditure amounts, etc. Every individual has a unique personal identification number. To protect privacy, data on patient identities were scrambled cryptographically in the NHIRD. All datasets can be interlinked through individual personal identification numbers. ICD-9-Clinical Modification (ICD-9-CM) was used to identify diagnoses.

Study participants We conducted a nationwide populationbased cohort study that included two groups. Children with EV infection (aged $<18$ years) during 2000-2007 were identified and followed up until 31 December 2008 or until first occurrence of type 1 diabetes. Individuals included in the EV infection group needed to have had at least two ambulatory claims or at least one inpatient claim during 2000-2007 (with the first diagnosis as an index date) using the following ICD-9-CM codes: 008.67 (enteritis due to enterovirus, $n=97$ ), 047 (meningitis due to enterovirus, $n=8198$ ), 047.0 (meningitis due to Coxsackievirus, $n=252$ ), 047.1 (meningitis due to echovirus, $n=22$ ), 048 (other enterovirus diseases of central nervous system, $n=509$ ), 074 (specific diseases due to Coxsackievirus, $n=5179$ ), 074.0 (herpangina, $n=436,372$ ), 074.1 (epidemic pleurodynia, $n=89$ ), 074.2 (Coxsackievirus carditis, $n=38$ ), 074.20 (Coxsackievirus carditis, unspecified, $n=18$ ), 074.21 (pericarditis due to Coxsackievirus, $n=1$ ), 074.23 (myocarditis due to Coxsackievirus, $n=31$ ), 074.3 (hand-foot-and-mouth disease, $n=116,441$ ), 074.8 (other specified diseases due to Coxsackievirus, $n=2043$ ), 079.1 (echovirus infection in conditions classified elsewhere and of unspecified site, $n=168$ ) or 079.2 (Coxsackievirus infection 
in conditions classified elsewhere and of unspecified site, $n=673$ ).

We then excluded 191 children with incomplete information on sex and residential area. A total of 576,967 children with EV infection were eligible. The group without EV infection comprised half of all insured children of the same age and without a diagnosis of $\mathrm{EV}$ infection. After excluding children infected with EV from 1996 to $2008(n=868,831)$, a total of $3,129,171$ children without EV were included. By use of frequency-matching with sex and birth year, children without EV infection were selected from those eligible. Further excluding children with a prior diagnosis of type 1 diabetes $(n=206)$ before the index date left 570,133 with and without EV infection in the final analysis (Fig. 1). The baseline or index date for the EV group was the first outpatient visit or inpatient admission for EV infection. For the non-EV group, an index date between 1 January 2000 and 31 December 2007 was randomly assigned according to the index date distribution of the EV group. This study was approved by the Ethical Review Board of China Medical University Hospital.

Outcome measures and comorbidities Follow-up personyears were derived for a child from index date to December 2008 or until onset of type 1 diabetes, death or withdrawal from the insurance system. The outcome event was type 1 diabetes (ICD-9-CM codes 250.x1, 250.x3). Children with atopic dermatitis (ICD-9-CM code 691.8), allergic rhinitis (ICD-9-CM code 477) or bronchial asthma (ICD-9-CM code 493) identified within 1 year of the index date were considered as having a comorbidity.
Statistical analysis The distribution of demographic data and comorbidities (age at entry, sex, atopic dermatitis, allergic rhinitis and bronchial asthma) were compared between groups. Differences were examined using the $\chi^{2}$ test for categorical variables and the $t$ test for continuous variables. Follow-up time served to estimate incidence rates of type 1 diabetes; Poisson regression was used to assess the incidence rate ratios of type 1 diabetes and the corresponding $95 \%$ CIs by comparing individuals with and without $\mathrm{EV}$ infection. Cumulative incidence of type 1 diabetes was computed by the Kaplan-Meier method, and differences in cumulative incidence between groups were tested using a logrank test. Cox proportional hazard regression models were used to assess the association between EV infection and type 1 diabetes, adjusting for potential confounders. Adjusted HRs and their $95 \%$ CIs were estimated. Interaction of EV infection status with age at entry, sex, atopic dermatitis, allergic rhinitis and bronchial asthma at baseline was examined by adding product terms into the full model, and the likelihood ratio was used to test for significance. SAS version 9.3 (SAS Institute, Cary, NC, USA) was used for data analyses; two-sided tests were performed, and $p<0.05$ was considered statistically significant.

\section{Results}

We identified 570,133 children with EV infection from 2000 2007 as the EV group and frequency-matched 570,133
Fig. 1 Flowchart of the recruitment procedure. T1DM, type 1 diabetes

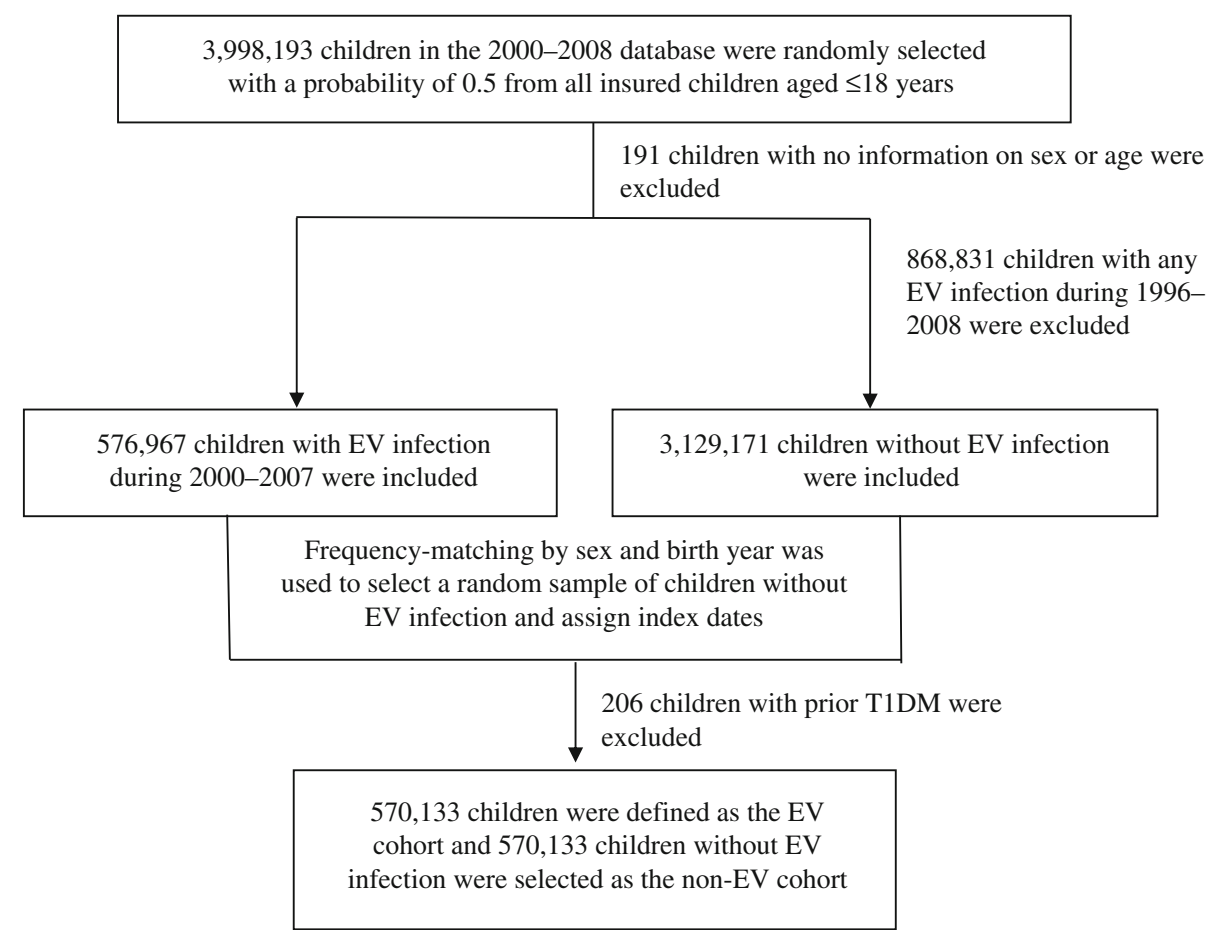


children without $\mathrm{EV}$ infection by sex and birth year as the nonEV group. Mean age at entry and sex distribution were similar in both groups. Compared with the non-EV group, the EV group had a significantly higher prevalence of atopic dermatitis, allergic rhinitis and bronchial asthma (Table 1). The incidence rate of type 1 diabetes was lower in the non-EV than the EV group (4 vs 6 per 100,000 person-years; incidence rate ratio 1.48 [95\% CI 1.19, 1.83]) (Table 2). The incidence rate of type 1 diabetes for both groups was further stratified based on age at entry, sex, allergic rhinitis and bronchial asthma. The incidence rate proved consistently lower for the non-EV than the EV group, except for children aged 10 years and over, as well as those with allergic rhinitis or bronchial asthma. Median follow-up time was 6.4 years overall. EV infection status was strongly associated with type 1 diabetes, exerting a $50 \%$ increased risk after 1 year of follow-up (logrank $p<0.001$, Fig. 2).

Multivariate-adjusted HRs also demonstrated that EV infection and older age at entry were associated with a higher risk of type 1 diabetes (Table 3). Compared with non-EV children, those in the EV group were more susceptible (HR 1.48 [95\% CI 1.19, 1.83]). Furthermore, children aged more than 10 years at entry showed elevated risk (HR 2.18 [95\% CI 1.18, 4.04]). The HRs of type 1 diabetes in children

Table 1 Sociodemographic factors and comorbidity according to EV status

\begin{tabular}{llll}
\hline Variable & \multicolumn{2}{l}{ EV infection status } & \multirow{2}{*}{$p$ value } \\
\cline { 2 - 3 } & No & Yes & $<0.001$ \\
\hline Age at entry (years) & & & \\
$\quad \leq 1$ & $42,252(7.41)$ & $35,380(6.21)$ & \\
$>1-3$ & $186,332(32.68)$ & $195,031(34.21)$ & \\
$>3-5$ & $165,609(29.05)$ & $165,659(29.06)$ & \\
$>5-10$ & $157,289(27.59)$ & $155,890(27.34)$ & \\
$>10$ & $18,651(3.27)$ & $18,173(3.19)$ & \\
Sex & & & $<00$ \\
$\quad$ Girl & $266,342(46.72)$ & $266,342(46.72)$ & \\
Boy & $303,791(53.28)$ & $303,791(53.28)$ & \\
Atopic dermatitis & & & $<0.001$ \\
$\quad$ No & $561,271(98.45)$ & $558,079(97.89)$ & \\
$\quad$ Yes & $8,862(1.55)$ & $12,054(2.11)$ & \\
Allergic rhinitis & & & $<0.001$ \\
No & $532,071(93.32)$ & $501,416(87.95)$ & \\
$\quad$ Yes & $38,062(6.68)$ & $68,717(12.05)$ & \\
Bronchial asthma & & & \\
No & $544,968(95.59)$ & $520,419(91.28)$ & \\
Yes & $25,165(4.41)$ & $49,714(8.72)$ & \\
\hline
\end{tabular}

$p$ values were calculated using the $\chi^{2}$ test

Data are presented as $n(\%)$

$n=1,140,266$ with allergic rhinitis, bronchial asthma or either one of these atopic diseases showed more variation than in those children without these diseases (Fig. 3): the 95\% CIs of HRs were greater in children with allergic rhinitis, bronchial asthma or either one of these atopic diseases than in corresponding groups without these diseases.

\section{Discussion}

The present study identifies an increased risk of type 1 diabetes among patients with EV infection. To the best of our knowledge, the study is the first nationwide retrospective cohort study on the association between type 1 diabetes and EV infection. The worldwide DIAMOND Project [4] and the EURODIAB [2] multicentre prospective registration study in Europe showed an accelerating epidemic of type 1 diabetes across ethnicities and geographic areas. This trend in different countries and among different ethnicities may be explained by genetic background, chemical exposure and/or infectious pathogens among persons with diverse demographic characteristics [35]. Genetic factors alone cannot explain this rise in type 1 diabetes.

The Environmental Determinants of Diabetes in the Young (TEDDY) [36] study, which is ongoing, aims to determine how environmental factors influence the onset of type 1 diabetes in susceptible patients. Existing findings from a large prospective birth cohort study have disclosed that Coxsackievirus B1 is associated with an increased risk of type 1 diabetes in Finland [13]. In addition, a virus antibody survey involving diverse European populations has suggested a link between Coxsackieviruses and type 1 diabetes [14]. In Finland, which has the highest incidence of childhood type 1 diabetes worldwide (64.3 per 100,000 person-years during 2006-2011) [6] and a low prevalence of EV infection [37], genetic background may play a major role [38, 39]. Hermann et al found that the $D Q B 1 * 0302 / * 0603$ genotype positively correlated with the risk of type 1 diabetes in Finland [40], whereas the $D Q B 1^{*} 0603$ genotype has been linked with protection from type 1 diabetes in populations outside Finland [41, 42].

Genetic predisposition may explain the high incidence of type 1 diabetes in Finland. However, in Asia, Africa and South America, which have a low but increasing incidence of type 1 diabetes $[5,43]$ and a high prevalence of $\mathrm{EV}$ infection, environmental factors such as EV infection may play a vital role [37]. In a Taiwanese study that estimated the average yearly crude incidence of type 1 diabetes in children [7], increasing incidence was reported among patients under 18 years of age, with an overall rate of 3.75 (boys 3.15 and girls 4.39) per 100,000 person-years during 1992-1996. Increasing incidence of type 1 diabetes as a result of decreasing incidence of EV infection has been hypothesised [44]. Taiwan has 
Table 2 Incidence rates and incidence rate ratios of type 1 diabetes according to EV status stratified by sociodemographic factors and comorbidity

\begin{tabular}{|c|c|c|c|c|c|c|c|c|}
\hline \multirow[t]{2}{*}{ Variable } & \multicolumn{3}{|c|}{ Without EV infection } & \multicolumn{3}{|c|}{ With EV infection } & \multirow[t]{2}{*}{ IRR } & \multirow[t]{2}{*}{$(95 \% \mathrm{CI})$} \\
\hline & No. of events & Person-years & IR & No. of events & Person-years & IR & & \\
\hline All & 141 & $3,627,392$ & 3.89 & 208 & $3,626,979$ & 5.73 & 1.48 & $(1.19,1.83)$ \\
\hline \multicolumn{9}{|c|}{ Age at entry (years) } \\
\hline$\leq 1$ & 7 & 294,658 & 2.38 & 15 & 253,019 & 5.93 & 2.50 & $(1.02,6.12)$ \\
\hline$>1-3$ & 40 & $1,245,225$ & 3.21 & 68 & $1,302,442$ & 5.22 & 1.63 & $(1.10,2.40)$ \\
\hline$>3-5$ & 46 & $1,047,282$ & 4.39 & 52 & $1,045,108$ & 4.98 & 1.13 & $(0.76,1.68)$ \\
\hline$>5-10$ & 38 & 935,671 & 4.06 & 64 & 924,243 & 6.92 & 1.71 & $(1.14,2.55)$ \\
\hline$>10$ & 10 & 104,557 & 9.56 & 9 & 102,166 & 8.81 & 0.92 & $(0.37,2.27)$ \\
\hline \multicolumn{9}{|l|}{ Sex } \\
\hline Girl & 61 & $1,684,340$ & 3.62 & 101 & $1,684,114$ & 6.00 & 1.66 & $(1.21,2.28)$ \\
\hline Boy & 80 & $1,943,052$ & 4.12 & 107 & $1,942,864$ & 5.51 & 1.34 & $(1.00,1.79)$ \\
\hline \multicolumn{9}{|c|}{ Allergic rhinitis } \\
\hline No & 130 & $3,415,308$ & 3.81 & 182 & $3,227,963$ & 5.64 & 1.48 & $(1.18,1.86)$ \\
\hline Yes & 11 & 212,084 & 5.19 & 26 & 399,016 & 6.52 & 1.26 & $(0.62,2.54)$ \\
\hline \multicolumn{9}{|c|}{ Bronchial asthma } \\
\hline No & 158 & $3,606,177$ & 4.38 & 227 & $3,358,232$ & 6.76 & 1.54 & $(1.26,1.89)$ \\
\hline Yes & 4 & 47,662 & 8.39 & 11 & 295,297 & 3.73 & 0.44 & $(0.14,1.39)$ \\
\hline
\end{tabular}

IR, incidence rate per 100,000 person-years

IRR, incidence rate ratio, compared with children without EV infection

relatively low type 1 diabetes incidence; we believe that the marked escalation of type 1 diabetes incidence in recent decades can be largely attributed to the highly endemic spread of EV infection in Taiwanese children, given that gene flow and genetic drift are likely to be fairly static over such a short period: the NHIRD dataset revealed a fluctuation in EV

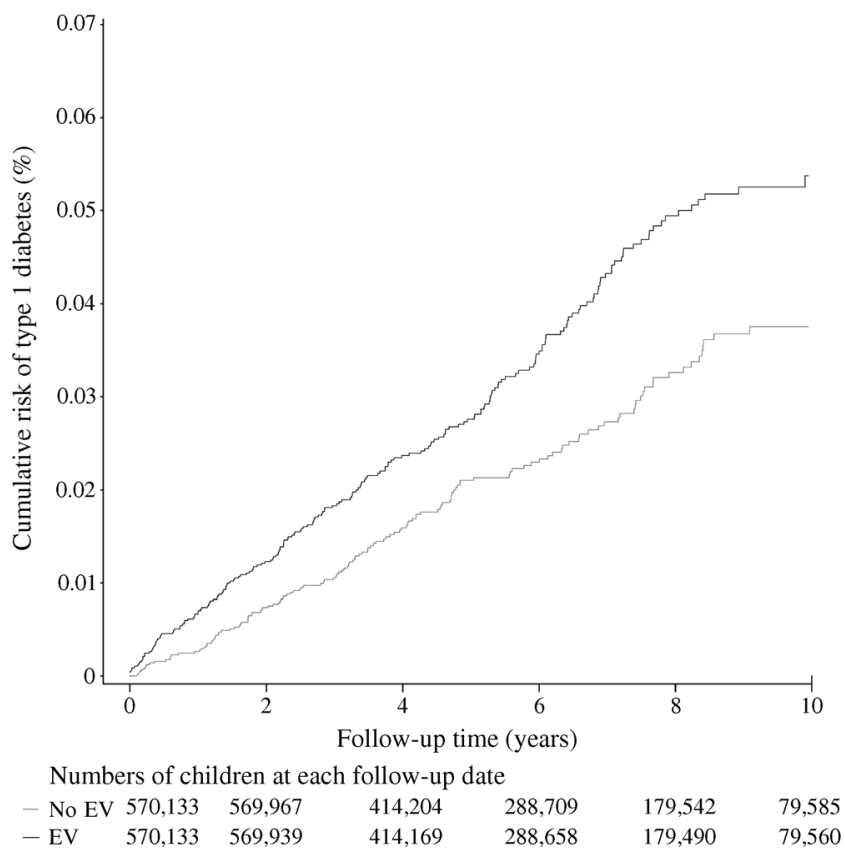

Fig. 2 Cumulative risk of type 1 diabetes based on EV infection status
Table 3 Crude and adjusted HRs of type 1 diabetes for EV infection, demographic characteristics and comorbidity

\begin{tabular}{|c|c|c|}
\hline Variable & Crude HR $(95 \% \mathrm{CI})$ & Adjusted HR $(95 \% \mathrm{CI})$ \\
\hline \multicolumn{3}{|c|}{ EV infection } \\
\hline No & 1.00 & 1.00 \\
\hline Yes & $1.48(1.19,1.83)^{* * *}$ & $1.48(1.19,1.83)^{* * *}$ \\
\hline \multicolumn{3}{|c|}{ Age (years) } \\
\hline$\leq 1$ & 1.00 & 1.00 \\
\hline$>1-3$ & $1.04(0.66,1.65)$ & $1.03(0.65,1.62)$ \\
\hline$>3-5$ & $1.14(0.72,1.81)$ & $1.13(0.71,1.79)$ \\
\hline$>5-10$ & $1.33(0.84,2.11)$ & $1.31(0.82,2.08)$ \\
\hline$>10$ & $2.24(1.21,4.15)^{*}$ & $2.18(1.18,4.04)^{*}$ \\
\hline \multicolumn{3}{|l|}{ Sex } \\
\hline Girl & 1.00 & 1.00 \\
\hline Boy & $1.00(0.81,1.24)$ & $1.01(0.82,1.24)$ \\
\hline \multicolumn{3}{|c|}{ Allergic rhinitis } \\
\hline No & 1.00 & 1.00 \\
\hline Yes & $1.27(0.90,1.79)$ & $1.30(0.90,1.87)$ \\
\hline \multicolumn{3}{|c|}{ Bronchial asthma } \\
\hline No & 1.00 & 1.00 \\
\hline Yes & $0.78(0.48,1.27)$ & $0.64(0.38,1.08)$ \\
\hline
\end{tabular}

Adjusted HR: each variable in the Cox proportional hazards regression, including EV infection, age, sex, allergic rhinitis and bronchial asthma, was adjusted for all variables in the model except itself

${ }^{*} p<0.05 ; * * * p<0.001$ 
Fig. 3 HRs of type 1 diabetes for EV infections stratified by allergic rhinitis, bronchial asthma or any one of these atopic diseases; $* * * p<0.001$

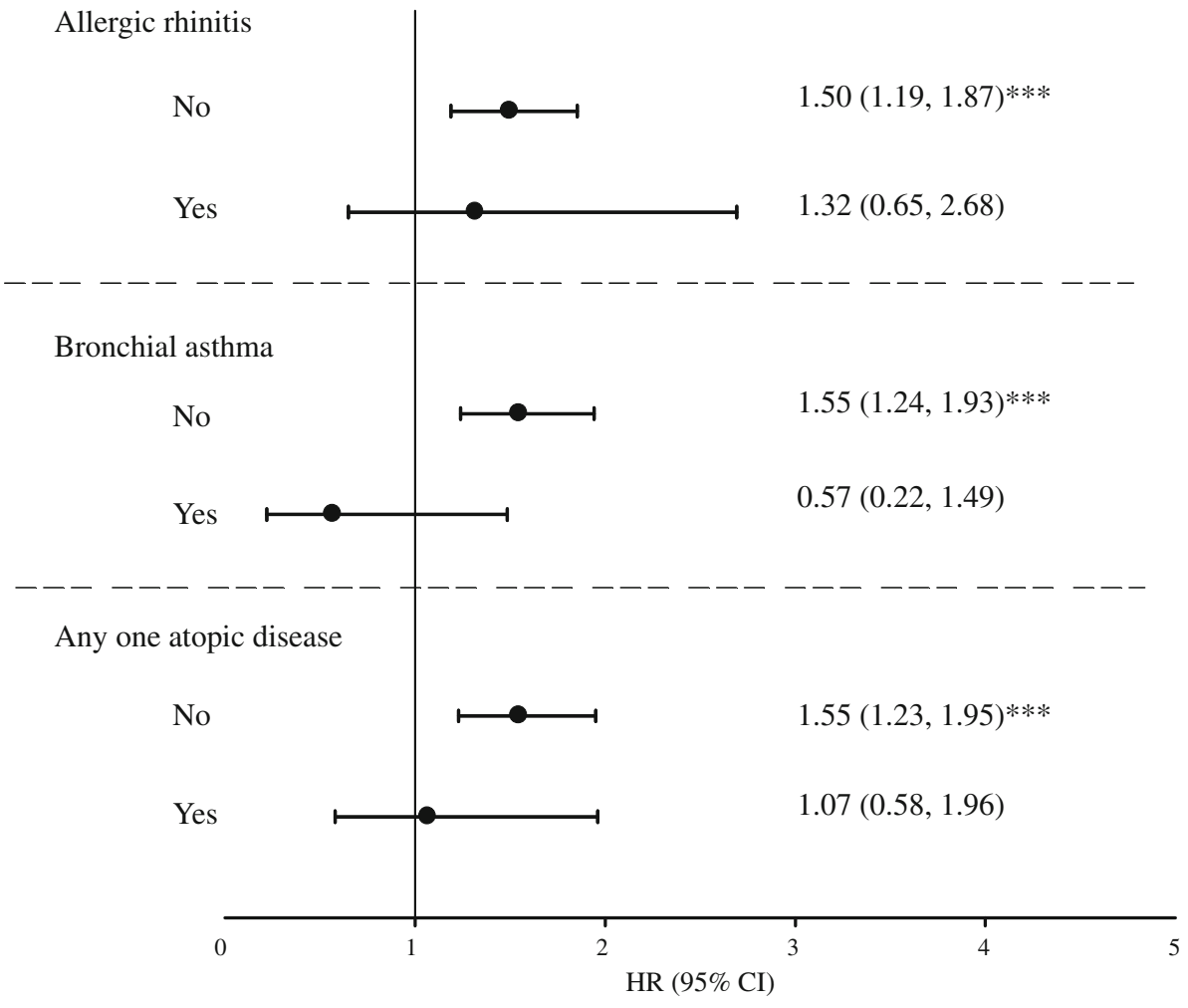

infection over 2000-2008 with an overall $10.5 \%$ increase from $1.24 \%$ in 2000 to $1.37 \%$ in 2008 , a continuing increase in incidence of EV infection.

Estimates of type 1 diabetes incidence in this study were consistent with those of earlier studies, indicating that peaks in the first presentation of type 1 diabetes appear in two age groups: 5-7 years and puberty [45]. The first peak may correspond to a time of frequent infection due to the beginning of school attendance, and the second with the pubertal growth spurt induced by gonadal steroids and increased pubertal growth hormone secretion that antagonises insulin [45].

We were also interested in connections between atopic disease (dermatitis, allergic rhinitis and bronchial asthma) and type 1 diabetes incidence. HRs showed an obvious trend toward lower risk in atopic than in non-atopic patients (Fig. 3). We postulated that in atopic groups, deviation of the immune system toward the $\mathrm{T}$ helper 2 pathway may confer protective effects that prevent these patients from developing type 1 diabetes (which is a T helper 1 pathway autoactivity disease) $[46,47]$. A case-control study has shown a negative correlation between childhood eczema and subsequent type 1 diabetes [33].

Animal studies have documented the concept of virusinduced diabetes, which increases biological plausibility of linkage between EV infection and type 1 diabetes. In 1968, Craighead and McLane reported that picornavirus could induce diabetes in adult mice [48]. Many studies since then have used rodent models of spontaneous diabetes, and much attention has been focused on EVs, especially Coxsackievirus B4 [15]. Moreover, concrete evidence illustrates a range of EVs infecting, replicating and lysing cultured human islets and insulin-producing cell lines in vitro [49], and markers of EV have also been found in the pancreas of patients with type 1 diabetes [24].

Advantages of our study merit attention. First, use of the administrative database avoided under-reporting of medical visits and hospitalisations [34]. Prior epidemiological and molecular studies were restricted by small sample sizes, even those from high-incidence countries, with no extensive epidemiological study design similar to ours. Second, the nationwide population-based study design prevented selection bias. Third, our study adjusted for confounding factors (age, sex and comorbidity). Whilst ethnicity was not included in adjustments, over $98 \%$ of Taiwanese people are of Han Chinese ethnicity; such a homogenous background cohort sharply lessens the likelihood of the results being confounded by ethnicity.

The current study has certain limitations. First, the precision of diagnoses based on ICD-9 obtained in administrative data described by doctors, without confirmation by virus culture, serology or molecular methods, might matter. Although the National Health Insurance programme regularly conducts expert reviews of patients' charts to randomly confirm claims from all hospitals, bias may arise from miscoding and misclassification. Second, laboratory confirmation or even information on the subtype of $\mathrm{EV}$ infection are not 
available from the database. Diagnoses of EV infections are based solely on clinical phenotypes. Clinical diagnoses of herpangina and hand-foot-and-mouth disease, which are easy to diagnose by phenotype, account for nearly $97 \%$ of EV infections. However, most EV infections present without clear symptoms, and these asymptomatic infections could not be identified in this study. Third, selecting patients younger than 18 years may underestimate the true numbers of patients with type 1 diabetes in Taiwan, as onset is not limited to this age bracket. Fourth, low incidence of type 1 diabetes coupled with small sample size for cases of atopic dermatitis, allergic rhinitis and bronchial asthma limits the ability to associate EV infection with type 1 diabetes under subgroups of such atopic diseases or with the rate effect modification of the association.

This nationwide retrospective cohort study found that type 1 diabetes is positively correlated with EV infection in patients younger than 18 years. Whilst the evidence of an association between EV infection and pathogenesis is observational, the conclusions are solid enough to guide further research on this association. The findings suggest that a vaccination strategy against EV infection might slow the rising incidence of type 1 diabetes.

Funding This study was supported primarily by the Bureau of National Health Insurance (DOH99-HP-1205) and Taiwan Ministry of Health and Welfare Clinical Trial and Research Center of Excellence (MOHW103TDU-B-212-113002).

Duality of interest The authors declare that there is no duality of interest associated with this manuscript.

Contribution statement CCL, TCL and HCL were responsible for the conception and design of the study. TCL and HCL acquired data and TCL analysed data. CHW, FJT, KPH, CCL and WC interpreted data. HCL and TCL were responsible for drafting the article. All authors revised the manuscript and approved the final version. TCL and HCL are responsible for the integrity of the work as a whole.

\section{References}

1. EURODIAB ACE Study Group (2000) Variation and trends in incidence of childhood diabetes in Europe. EURODIAB ACE Study Group. Lancet 355:873-876

2. Patterson CC, Dahlquist GG, Gyurus E, Green A, Soltesz G (2009) Incidence trends for childhood type 1 diabetes in Europe during 1989-2003 and predicted new cases 2005-20: a multicentre prospective registration study. Lancet 373:2027-2033

3. Chong JW, Craig ME, Cameron FJ et al (2007) Marked increase in type 1 diabetes mellitus incidence in children aged $0-14$ yr in Victoria, Australia, from 1999 to 2002. Pediatr Diabetes 8:67-73

4. The DIAMOND Project Group (2006) Incidence and trends of childhood type 1 diabetes worldwide 1990-1999. Diabet Med 23: 857-866

5. Patterson CC, Gyurus E, Rosenbauer J et al (2012) Trends in childhood type 1 diabetes incidence in Europe during 1989-2008: evidence of non-uniformity over time in rates of increase. Diabetologia 55:2142-2147

6. Harjutsalo V, Sund R, Knip M, Groop P-H (2013) Incidence of type 1 diabetes in Finland letters. JAMA 310:427-428

7. Tseng CH (2008) Incidence of type 1 diabetes mellitus in children aged 0-14 years during 1992-1996 in Taiwan. Acta Paediatr 97:392393

8. Hober D, Sauter P (2010) Pathogenesis of type 1 diabetes mellitus: interplay between enterovirus and host. Nat Rev Endocrinol 6:279289

9. Tauriainen S, Oikarinen S, Oikarinen M, Hyoty H (2011) Enteroviruses in the pathogenesis of type 1 diabetes. Semin Immunopathol 33:4555

10. Yeung WC, Rawlinson WD, Craig ME (2011) Enterovirus infection and type 1 diabetes mellitus: systematic review and meta-analysis of observational molecular studies. BMJ 342:d35

11. Gamble DR, Kinsley ML, FitzGerald MG, Bolton R, Taylor KW (1969) Viral antibodies in diabetes mellitus. Br Med J 3:627-630

12. Gamble DR, Taylor KW (1969) Seasonal incidence of diabetes mellitus. Br Med J 3:631-633

13. Laitinen OH, Honkanen H, Pakkanen O et al (2014) Coxsackievirus $\mathrm{B} 1$ is associated with induction of beta-cell autoimmunity that portends type 1 diabetes. Diabetes 63:446-455

14. Oikarinen S, Tauriainen S, Hober D et al (2014) Virus antibody survey in different European populations indicates risk association between coxsackievirus B1 and type 1 diabetes. Diabetes 63:655-662

15. Jaidane H, Hober D (2008) Role of coxsackievirus B4 in the pathogenesis of type 1 diabetes. Diabetes Metab 34:537-548

16. Frisk G, Nilsson E, Tuvemo T, Friman G, Diderholm H (1992) The possible role of Coxsackie A and echo viruses in the pathogenesis of type I diabetes mellitus studied by IgM analysis. J Infect 24:13-22

17. Helfand RF, Gary HE Jr, Freeman CY, Anderson LJ, Pallansch MA (1995) Serologic evidence of an association between enteroviruses and the onset of type 1 diabetes mellitus. Pittsburgh Diabetes Research Group. J Infect Dis 172:1206-1211

18. Roivainen M, Knip M, Hyoty H et al (1998) Several different enterovirus serotypes can be associated with prediabetic autoimmune episodes and onset of overt IDDM. Childhood Diabetes in Finland (DiMe) Study Group. J Med Virol 56:74-78

19. Salvatoni A, Baj A, Bianchi G, Federico G, Colombo M, Toniolo A (2013) Intrafamilial spread of enterovirus infections at the clinical onset of type 1 diabetes. Pediatr Diabetes 14:407-416

20. Gleason RE, Kahn CB, Funk IB, Craighead JE (1982) Seasonal incidence of insulin-dependent diabetes (IDDM) in Massachusetts, 1964-1973. Int J Epidemiol 11:39-45

21. Huff JC, Hierholzer JC, Farris WA (1974) An “outbreak" of juvenile diabetes mellitus: consideration of a viral etiology. Am J Epidemiol 100:277-287

22. Oikarinen S, Martiskainen M, Tauriainen S et al (2011) Enterovirus RNA in blood is linked to the development of type 1 diabetes. Diabetes 60:276-279

23. Richardson SJ, Leete P, Bone AJ, Foulis AK, Morgan NG (2013) Expression of the enteroviral capsid protein VP1 in the islet cells of patients with type 1 diabetes is associated with induction of protein kinase R and downregulation of Mcl-1. Diabetologia 56:185-193

24. Willcox A, Richardson SJ, Bone AJ, Foulis AK, Morgan NG (2011) Immunohistochemical analysis of the relationship between islet cell proliferation and the production of the enteroviral capsid protein, VP1, in the islets of patients with recent-onset type 1 diabetes. Diabetologia 54:2417-2420

25. Krogvold L, Edwin B, Buanes T et al (2014) Pancreatic biopsy by minimal tail resection in live adult patients at the onset of type 1 diabetes: experiences from the DiViD study. Diabetologia 57:841-843

26. Campbell-Thompson ML, Atkinson MA, Butler AE et al (2013) The diagnosis of insulitis in human type 1 diabetes. Diabetologia 56: $2541-2543$ 
27. Oikarinen M, Tauriainen S, Oikarinen S et al (2012) Type 1 diabetes is associated with enterovirus infection in gut mucosa. Diabetes 61: 687-691

28. Campbell-Thompson M, Wasserfall C, Kaddis J et al (2012) Network for Pancreatic Organ Donors with Diabetes (nPOD): developing a tissue biobank for type 1 diabetes. Diabetes Metab Res Rev 28:608617

29. Sarmiento L, Cabrera-Rode E, Lekuleni L et al (2007) Occurrence of enterovirus RNA in serum of children with newly diagnosed type 1 diabetes and islet cell autoantibody-positive subjects in a population with a low incidence of type 1 diabetes. Autoimmunity 40:540-545

30. Craig ME, Howard NJ, Silink M, Rawlinson WD (2003) Reduced frequency of HLA DRB1*03-DQB1*02 in children with type 1 diabetes associated with enterovirus RNA. J Infect Dis 187:15621570

31. Maha MM, Ali MA, Abdel-Rehim SE, Abu-Shady EA, El-Naggar BM, Maha YZ (2003) The role of coxsackieviruses infection in the children of insulin dependent diabetes mellitus. J Egypt Public Health Assoc 78: 305-318

32. Kawashima H, Ihara T, Ioi $\mathrm{H}$ et al (2004) Enterovirus-related type 1 diabetes mellitus and antibodies to glutamic acid decarboxylase in Japan. J Infect 49:147-151

33. Stene LC, Ronningen KS, Bjornvold M, Undlien DE, Joner G (2010) An inverse association between history of childhood eczema and subsequent risk of type 1 diabetes that is not likely to be explained by HLA-DQ, PTPN22, or CTLA4 polymorphisms. Pediatr Diabetes 11:386-393

34. Lu JF, Hsiao WC (2003) Does universal health insurance make health care unaffordable? Lessons from Taiwan. Health Aff (Millwood) 22: $77-88$

35. Newhook LA, Penney S, Fiander J, Dowden J (2012) Recent incidence of type 1 diabetes mellitus in children $0-14$ years in Newfoundland and Labrador, Canada climbs to over 45/100,000: a retrospective time trend study. BMC Res Notes 5:628

36. TEDDY Study Group (2008) The Environmental Determinants of Diabetes in the Young (TEDDY) study. Ann N Y Acad Sci 1150:113
37. Viskari H, Ludvigsson J, Uibo R et al (2004) Relationship between the incidence of type 1 diabetes and enterovirus infections in different European populations: results from the EPIVIR project. J Med Virol 72:610-617

38. Noble JA, Erlich HA (2012) Genetics of type 1 diabetes. Cold Spring Harb Perspect Med 2:a007732

39. Stankov K, Benc D, Draskovic D (2013) Genetic and epigenetic factors in etiology of diabetes mellitus type 1. Pediatrics 132:11121122

40. Hermann R, Turpeinen H, Laine AP et al (2003) HLA DR-DQ-encoded genetic determinants of childhood-onset type 1 diabetes in Finland: an analysis of 622 nuclear families. Tissue Antigens 62:162-169

41. Pugliese A, Gianani R, Moromisato R et al (1995) HLADQB1*0602 is associated with dominant protection from diabetes even among islet cell antibody-positive first-degree relatives of patients with IDDM. Diabetes 44:608-613

42. Sanjeevi CB (2000) HLA-DQ6-mediated protection in IDDM. Hum Immunol 61:148-153

43. Stanescu DE, Lord K, Lipman TH (2012) The epidemiology of type 1 diabetes in children. Endocrinol Metab Clin N Am 41:679-694

44. Viskari HR, Koskela P, Lonnrot M et al (2000) Can enterovirus infections explain the increasing incidence of type 1 diabetes? Diabetes Care 23:414-416

45. Achenbach P, Bonifacio E, Koczwara K, Ziegler AG (2005) Natural history of type 1 diabetes. Diabetes 54(Suppl 2):S25-S31

46. Cakir M, Akcay S, Karakas T, Gedik Y, Okten A, Orhan F (2008) Prevalence of atopy in children with type 1 diabetes mellitus, hepatitis B virus carriers, and healthy children: role of $\mathrm{T}$ helper 1 (Th1)-type immune response. Allergy Asthma Proc 29:166-170

47. Tzeng ST, Hsu SG, Fu LS, Chi CS (2007) Prevalence of atopy in children with type 1 diabetes mellitus in central Taiwan. J Microbiol Immunol Infect 40:74-78

48. Craighead JE, McLane MF (1968) Diabetes mellitus: induction in mice by encephalomyocarditis virus. Science 162:913-914

49. Ylipaasto P, Smura T, Gopalacharyulu P et al (2012) Enterovirusinduced gene expression profile is critical for human pancreatic islet destruction. Diabetologia 55:3273-3283 